\title{
Eigenfunctions of Two-Scale Difference Equations and Appell Polynomials
}

\author{
L. Berg and M. Krüppel
}

\begin{abstract}
Both classical and distributional solutions of two-scale difference equations are interpreted as eigenfunctions, which are closely connected with Appell polynomials. Different generating functions are analyzed and the relations between them. Equivalent eigenfunctions as well as equivalent and minimal characteristic polynomials are defined and investigated in detail via the rational solution of a basic functional equation. Finally, reversed eigenfunctions are introduced and characterized.

Keywords: Two-scale difference equations, distributional solutions, eigenvalue problems, Appell polynomials, basic functional equation, equivalent and reversed eigenfunctions, minimal characteristic polynomials, sums of shifted eigenfunctions
\end{abstract}

AMS subject classification: 39A10, 39B32, 46F10, 47A75, 26C05, 33C65

\section{Preliminaries}

A functional equation of the form

$$
\lambda \varphi\left(\frac{t}{2}\right)=\sum_{\nu=0}^{N} c_{\nu} \varphi(t-\nu) \quad(t \in \mathbb{R})
$$

with $N \in \mathbb{N}_{0}$, and complex coefficients where $\lambda \neq 0$ and $c_{0} c_{N} \neq 0$, is called a two-scale difference equation. Such equations are intensively investigated and applied in wavelet theory, multiresolution analysis and subdivision schemes (cf. [9-11]). Throughout this paper we use the notation

$$
P(w)=\sum_{\nu=0}^{N} c_{\nu} w^{\nu}
$$

for the characteristic polynomial, where $P(0) \neq 0$ according to $c_{0} \neq 0$, and we always use the normalization

$$
\sum_{\nu=0}^{N} c_{\nu}=1
$$

Both authors: FB Math. der Universität, Universitätspl. 1, D-18051 Rostock

lothar.berg@mathematik.uni-rostock.de

manfred.krueppel@mathematik.uni-rostock.de 
so that $P(1)=1$ according to (1.3). The characteristic polynomial $P$ is closely connected with the so-called mask or symbol of (1.1), which is equal to $\frac{1}{2 \lambda} P$. For $\lambda=\frac{1}{2}$ all three terms coincide.

Our aim is to investigate non-trivial solutions of (1.1) in the class of generalized functions consisting of complex valued continuous functions and their derivatives of finite order in the distributional sense [1] and to interpret these solutions as eigenfunctions. For this reason we first introduce the

Definition 1.1. A generalized function $f$ is called a comparison function if it satisfies the two "boundary" conditions:

(i) $f(t)=0$ for $t<0$.

(ii) $f(t)$ is equal to a polynomial for great $t$.

If $f$ is a comparison function, then $f^{(n)}(n \in \mathbb{Z})$ is also a comparison function where $f^{(n)}$ denotes for $n \geq 0$ as usual the derivative of order $n$ and for $n<0$ the corresponding iterated integral of order $-n$ subject to (i). For later purposes it is crucial to sharpen (ii) by the requirement that there exists a certain $n \in \mathbb{Z}$ such that

(iii) $f^{(n)}(t)=1$ for great $t$.

This is a normalization which means that the polynomials in (ii) have the main term $\frac{1}{n !} t^{n}$ in the case $n \in \mathbb{N}_{0}$. The set of all comparison functions satisfying (iii) for a fixed $n$ shall be denoted by $\mathcal{F}_{n}$. Obviously, if $f_{m} \in \mathcal{F}_{m}$ for a fixed $m \in \mathbb{Z}$, then

$$
f_{n}=f_{m}^{(m-n)}
$$

belongs to $\mathcal{F}_{n}$ for arbitrary $n \in \mathbb{Z}$.

For comparison functions with property (1.4) we introduce the further

Definition 1.2. A sequence $f_{n} \in \mathcal{F}_{n}(n \in \mathbb{Z})$ satisfying (1.4) for arbitrary $m, n \in \mathbb{Z}$ is called a comparison sequence.

Condition (1.4) means that every function $f_{m} \in \mathcal{F}_{m}$ generates a comparison sequence and that different terms of a comparison sequence arise from each other by successive integration or differentiation.

Every element $f_{n} \in \mathcal{F}_{n}(n \in \mathbb{Z})$ possesses a Laplace transform $\mathcal{L}\left\{f_{n}\right\}$ (cf. [2]). In the case $n<0, f_{n}$ is compactly supported (since the polynomials in (ii) vanish), and $\mathcal{L}\left\{f_{n}\right\}$ is an entire function of $z$, where $z$ is the complex variable of the Laplace transform. The differentiation and the integration theorem show for a comparison sequence that the products

$$
z^{n+1} \mathcal{L}\left\{f_{n}\right\}=\mathcal{L}\left\{f_{-1}\right\}
$$

are independent of $n$ for all $n \in \mathbb{Z}$, and therefore also entire functions. Let us mention that, after multiplication with a suitable factor, every non-trivial compactly supported generalized function $f$ belongs to a certain $\mathcal{F}_{n}$ with $n<1$. Otherwise, all integrals of $f$ would also be compactly supported, and $\mathcal{L}\{f\}$ would have the zero $z=0$ with an infinite order, which is impossible.

After these preparations, we come back to two-scale difference equations. 
Definition 1.3. The non-trivial solutions of (1.1) which are simultaneously comparison functions, are called eigenfunctions. The normalized eigenfunction belonging to $\mathcal{F}_{n}$ is denoted by $\varphi_{n}$ and the corresponding eigenvalue by $\lambda_{n}$.

It is well known that the eigenvalues are exactly the numbers $\lambda_{n}=2^{n}(n \in \mathbb{Z})$ and that they all are simple (cf. $[7,12,13])$. The eigenfunctions $\varphi_{n}$ are continuous for all $t \in \mathbb{R}$, if $n$ is sufficiently great, and proper distributions, if $-n$ is sufficiently great. In the case $n<0$ the support of $\varphi_{n}$ is contained in $[0, N]$ (cf. [11]). A Lebesgue-integrable compactly supported solution of (1.1) with the usual normalization

$$
\int_{0}^{N} \varphi(t) d t=1
$$

is the eigenfunction $\varphi=\varphi_{-1}$, i.e. it must be $\lambda=\frac{1}{2}$ in (1.1). This is a consequence of our normalization (iii) which together with $\lambda_{n}=2^{n}$ also implies that the eigenfunctions $\varphi_{n}$ form a comparison sequence. From this and the support property mentioned before it follows that it is the domain $t>N$ in which the eigenfunctions $\varphi_{n}$ are equal to polynomials subject to (ii), and that these polynomials are Appell polynomials generated by the Laplace transform of $\varphi_{-1}$ (cf. [4]). From [7: Theorem 6.1] it is known that the natural extension of these polynomials to all $t \in \mathbb{R}$ are likewise solutions of (1.1) with $\lambda=2^{n}$ (but of course no eigenfunctions).

In the sequel we investigate first some properties of comparison sequences in connection with Appell polynomials, which are needed later on in their specialization to eigenfunctions. The main goal of this paper is to compare the two-scale difference equation (1.1) with a second one

$$
\tilde{\lambda} \tilde{\varphi}\left(\frac{t}{2}\right)=\sum_{\nu=0}^{\tilde{N}} \tilde{c}_{\nu} \tilde{\varphi}(t-\nu)
$$

and to clarify the conditions such that corresponding eigenfunctions $\varphi_{n}, \tilde{\varphi}_{m}(n, m \in \mathbb{Z})$ satisfy relations of the form

$$
\varphi_{n}(t)=\sum_{k=0}^{\infty} r_{k} \tilde{\varphi}_{m}(t-k) \quad(t \in \mathbb{R})
$$

which up to now were only known in special cases (cf. [5, 6, 10, 14]), and in particular for cardinal splines (cf. [17, 20]). We introduce the concept of equivalent eigenfunctions $\varphi_{n} \sim \tilde{\varphi}_{m}$ and equivalent characteristic polynomials $P \sim \tilde{P}$ and give a complete characterization of these equivalences by means of our basic functional equation and its so-called canonical solution. Moreover, we determine the minimal characteristic polynomial within the class of equivalent characteristic polynomials which permits to survey the entire class. These results generalize investigations in [10: Section 5]. As application we deal with the relation

$$
\sum_{\nu=0}^{N} \varphi_{-1}(t+\nu)=1 \quad(-1<t<1),
$$


which is known for Lebesgue-integrable $\varphi_{-1}$ (cf. $[5,19]$ ). We show that a condition of [5], which is necessary for the Lebesgue-integrability of $\varphi_{-1}$, is necessary and sufficient for the validity of (1.9) in the general distributional case. A necessary and sufficient condition of [16] for the linear independence of shifts of $\varphi_{-1}$ is simplified by means of the minimal characteristic polynomial. Another application deals with infinite products and with power series containing the binary sum-of-digits function. Finally, reversed eigenfunctions are introduced by means of a so-called Appell triple, and their properties are studied.

\section{Comparison sequences and Appell polynomials}

In this section we are going to point out the connection between comparison sequences and Appell polynomials (cf. [8]). Given a (formal) power series

$$
F(z)=\sum_{n=0}^{\infty} a_{n} z^{n}
$$

with complex $a_{n}$, Appell polynomials $p_{n}\left(n \in \mathbb{N}_{0}\right)$ can be defined as coefficients of the generating function

$$
e^{t z} F(z)=\sum_{n=0}^{\infty} p_{n}(t) z^{n} \quad(t \in \mathbb{R}),
$$

i.e. explicitly by means of the representation

$$
p_{n}(t)=\sum_{k=0}^{n} \frac{a_{n-k}}{k !} t^{k} .
$$

They also can be defined recursively by one of the recursions

$$
p_{n}(t+\tau)=\sum_{k=0}^{n} \frac{1}{k !} p_{n-k}(\tau) t^{k} \quad(t, \tau \in \mathbb{R})
$$

and

$$
p_{n+1}^{\prime}(t)=p_{n}(t)
$$

for $n \in \mathbb{N}_{0}$, where in the last case one has to start with a constant polynomial $p_{0}=a_{0}$. For convenience we extend the definition of Appell polynomials by $p_{-n}(t)=0$ for $n \in \mathbb{N}$. Replacing $t$ in (2.2) by $t+s$ with a fixed parameter $s \in \mathbb{R}$, we get

$$
e^{t z} e^{s z} F(z)=\sum_{n=0}^{\infty} p_{n}(t+s) z^{n}
$$

and introducing the series

$$
e^{s z} F(z)=\sum_{n=0}^{\infty} b_{n} z^{n}
$$


where $b_{n}$ depends on $s$, we obtain by means of $(2.1)-(2.3)$

$$
p_{n}(t+s)=\sum_{k=0}^{n} \frac{b_{n-k}}{k !} t^{k}
$$

For $t=s, \tau=-s$ and $t=s, \tau=0$, respectively, equation (2.4) implies the following equations between the coefficients $a_{n}=p_{n}(0)$ and $b_{n}=p_{n}(s)$ :

$$
\left.\begin{array}{l}
a_{n}=\sum_{k=0}^{n} \frac{b_{n-k}}{k !}(-s)^{k} \\
b_{n}=\sum_{k=0}^{n} \frac{a_{n-k}}{k !} s^{k}
\end{array}\right\} .
$$

In particular, we have $p_{0}(t)=a_{0}=b_{0}$.

Comparing (2.5) with the property (1.4) in the case $m=n+1$ and taking into account the boundary condition (ii), we see that comparison sequences are Appell polynomials for great $t$. More precisely:

Proposition 2.1. Assume that $f_{n}(n \in \mathbb{Z})$ is a comparison sequence and assume that $p_{n}$ are the Appell polynomials corresponding to the entire function

$$
F(z)=\mathcal{L}\left\{f_{-1}\right\}
$$

Then for all $s$ with supp $f_{-1} \subseteq[0, s]$ we have

$$
f_{n}(t)=p_{n}(t) \quad(t>s)
$$

and for $n \in \mathbb{N}_{0}$ the representation

$$
p_{n}(t)=\frac{1}{n !} \int_{0}^{s}(t-\tau)^{n} f_{-1}(\tau) d \tau
$$

holds.

Proof. In the case that $f_{-1}$ is Lebesgue-integrable the proposition was already proved in [4: Proposition 4.1] with $N=s$ and $g_{0}=f_{-1}$. By partial integration representation $(2.12)$ can be transformed into

$$
p_{n}(t)=\frac{1}{(n-m-1) !} \int_{0}^{s}(t-s)^{n-m-1} f_{m}(\tau) d \tau+\sum_{\nu=0}^{m} \frac{b_{\nu}}{(n-\nu) !}(t-s)^{n-\nu}
$$

$\left(m \in \mathbb{N}_{0}\right)$ with $b_{\nu}$ from $(2.7)$ and vanishing integral for $m \geq n$. In the case that only $f_{m}$ is locally Lebesgue-integrable, but $f_{-1}$ a proper distribution, representation (2.13) is the classical interpretation of (2.12) and can be used in connection with $F(z)=z^{m+1} \mathcal{L}\left\{f_{m}\right\}$ (see $(1.5)$ ), to prove the proposition analogously as it was pointed out in [4: Corollary 4.1], for the case $m=0$ 
Example 2.2 (Sums). Let $f_{n}(n \in \mathbb{Z})$ be a comparison sequence and let $f_{-1}$ have the property

$$
\sum_{k=0}^{\infty} f_{-1}(t-k)=1 \quad(t>s)
$$

with a certain $s>0$. In particular, this relation is satisfied for $s=N$, if $f_{-1}=\varphi_{-1}$ is a Lebesgue-integrable eigenfunction (cf. [5, 19]). The left-hand side of $(2.14)$ belongs to $\mathcal{F}_{0}$. Hence we can denote it by $\tilde{f}_{0}$ and construct the corresponding comparison sequence $\tilde{f}_{n}$. Since

$$
\mathcal{L}\left\{\tilde{f}_{-1}\right\}=\frac{1}{1-e^{-z}} z F(z)=\frac{z e^{z}}{e^{z}-1} F(z)
$$

with (2.10), application of Proposition 2.1 to $\tilde{f}_{n}$ instead of $f_{n}$ yields

$$
\sum_{k=0}^{\infty} f_{n-1}(t-k)=\sum_{\nu=0}^{n} \frac{a_{n-\nu}}{\nu !} B_{\nu}(t+1)
$$

for $n \in \mathbb{N}_{0}$ and $t>s$, where $B_{\nu}(\cdot)$ are the Bernoulli polynomials and $a_{n}$ the coefficients of $F(z)=\mathcal{L}\left\{f_{-1}\right\}$ in $(2.1)$.

Example 2.3 (Convolutions). Let $f_{n}, \tilde{f}_{n}$ be two comparison sequences and consider the convolution

$$
f_{n} * \tilde{f}_{m}=\int_{0}^{t} f_{n}(t-\tau) \tilde{f}_{m}(\tau) d \tau
$$

$(n, m \in \mathbb{Z})$. For locally Lebesgue-integrable functions $f_{n}, \tilde{f}_{m}$ it can easily be seen that $f_{n} * \tilde{f}_{m} \in \mathcal{F}_{n+m+1}$, and differentiation shows that this is also valid for distributions. Using suggestive notations and considering $\operatorname{supp}\left(f_{-1} * \tilde{f}_{-1}\right) \subseteq[0, s+\tilde{s}]$,

$$
\mathcal{L}\left\{f_{-1} * \tilde{f}_{-1}\right\}=F(z) \tilde{F}(z)
$$

as well as (2.12), application of Proposition 2.1 to $f_{n} * \tilde{f}_{-1}$ instead of $f_{n}$ yields

$$
f_{n} * \tilde{f}_{-1}=\sum_{\nu=0}^{n} p_{\nu}(t) \tilde{a}_{n-\nu}=\sum_{\nu=0}^{n} \frac{\tilde{a}_{n-\nu}}{\nu !} \int_{0}^{s}(t-\tau)^{\nu} f_{-1}(\tau) d \tau
$$

for $t>s+\tilde{s}\left(n \in \mathbb{N}_{0}\right)$, and for the same $t$ and $n$ by means of (2.3) and (2.12)

$$
f_{n} * \tilde{f}_{-1}=\frac{1}{n !} \int_{0}^{s} \int_{0}^{\tilde{s}}(t-\tau-\sigma)^{n} f_{-1}(\tau) \tilde{f}_{-1}(\sigma) d \tau d \sigma .
$$

For $n+m \geq-1$, the general case $(2.16)$ can be reduced to $(2.17)$ by means of $f_{n} * \tilde{f}_{m}=$ $f_{n+m+1} * \tilde{\tilde{f}}_{-1}$.

Example 2.4 (Polynomials). Let $F$ in (2.10) be a polynomial $F(z)=\sum_{k=0}^{K} a_{k} z^{k}$ with $F(0)=1$. Then $f_{-1}=\sum_{k=0}^{K} a_{k} \delta^{(k)}$ and $f_{n}=p_{n} h$ for $n \geq m$, where $h$ denotes Heaviside's jump function, $\delta=h^{\prime}$ Dirac's distribution and where $p_{n}$ is defined by (2.3) with $a_{k}=0$ for $k>K$.

In particular, Proposition 2.1 can be applied to the eigenfunctions $\varphi_{n}$ of $(1.1)$ instead of $f_{n}$, so that according to $s \geq N$ we obtain: 
Corollary 2.5. For $t>N$ the eigenfunctions $\varphi_{n}$ of (1.1) belonging to the eigenvalues $\lambda_{n}=2^{n}$ are equal to the Appell polynomials corresponding to the entire function $F(z)=\phi(z)$ with

$$
\phi(z)=\mathcal{L}\left\{\varphi_{-1}\right\},
$$

i.e. $\varphi_{n}(t)=p_{n}(t)$ for $t>N$ and all $n \in \mathbb{Z}$.

For eigenfunctions $\varphi_{n}$ and $\tilde{\varphi}_{m}$ it is well known that the convolution $\varphi_{n} * \tilde{\varphi}_{m}$ is a solution of (1.1) with the characteristic polynomial $P \tilde{P}$ to the eigenvalue $\lambda=2^{n+m+1}$ (cf. [9: p. 26]), but Example 2.3 shows that this convolution is also a normalized eigenfunction.

Applying the Laplace transform to (1.1) it follows that (2.18) satisfies the functional equation

$$
\phi(2 z)=P\left(e^{-z}\right) \phi(z)
$$

where $P$ is the characteristic polynomial (1.2) with $P(1)=1$, and that (2.18) possesses the representation

$$
\phi(z)=\prod_{j=1}^{\infty} P\left(e^{-z / 2^{j}}\right)
$$

for all $z \in \mathbb{C}$ (cf. [11: p. 175]). Note that $\phi(0)=1$, and that this value is equivalent to $\varphi_{0}(t)=1$ for $t>N$.

Proposition 2.6. The coefficients in (2.1) of the function $F(z)=\phi(z)$ from (2.18) can be calculated recursively by

$$
\left.\begin{array}{l}
a_{0}=1 \\
a_{n}=\frac{1}{2^{n}-1} \sum_{k=1}^{n}(-1)^{k} \frac{a_{n-k}}{k !} \sum_{\nu=1}^{N} \nu^{k} c_{\nu} \quad(n \in \mathbb{N})
\end{array}\right\} .
$$

Proof. First, (2.20) implies $a_{0}=\phi(0)=1$ in view of $P(1)=1$. According to (2.19) we obtain

$$
\sum_{n=0}^{\infty} a_{n}(2 z)^{n}=P\left(e^{-z}\right) \sum_{n=0}^{\infty} a_{n} z^{n}=\sum_{\nu=0}^{N} c_{\nu} e^{-\nu z} \sum_{\mu=0}^{\infty} a_{\mu} z^{\mu} .
$$

Using the Taylor series for the exponential function and comparing the coefficients of $z^{n}$, we get

$$
2^{n} a_{n}=\sum_{k=0}^{n} a_{n-k} \frac{1}{k !} \sum_{\nu=0}^{N}(-\nu)^{k} c_{\nu} .
$$

In view of $P(1)=1$ this equation is an identity for $n=0$, and for $n>0$ it yields the recursion $(2.21)$

\section{Remark 2.7.}

1. According to (2.21) the coefficients $a_{n}$ can also be expressed directly by $P$, e.g.

$$
\left.\begin{array}{l}
a_{1}=-P^{\prime}(1) \\
a_{2}=\frac{1}{3} P^{\prime}(1)^{2}+\frac{1}{6}\left(P^{\prime}(1)+P^{\prime \prime}(1)\right)
\end{array}\right\} .
$$


2. Using the fact that the polynomials $p_{n}$ are solutions of (1.1) with $\lambda=2^{n}$ for $t \in \mathbb{R}$, their coefficients in (2.3) were already set up in [7], however with another notation and another normalization.

3. Analogously as in (2.21), the coefficients $b_{n}=p_{n}(N)$ can be calculated recursively:

$$
\left.\begin{array}{l}
b_{0}=1 \\
b_{n}=\frac{1}{2^{n}-1} \sum_{k=1}^{n} \frac{b_{n-k}}{k !} \sum_{\ell=1}^{N} \ell^{k} c_{N-\ell} \quad(n \in \mathbb{N})
\end{array}\right\} .
$$

Namely, by means of (2.7) with $s=N$ and (2.19) we also can derive the equation

$$
\sum_{n=0}^{\infty} b_{n}(2 z)^{n}=e^{N z} P\left(e^{-z}\right) \sum_{n=0}^{\infty} b_{n} z^{n}=\sum_{\nu=0}^{N} c_{\nu} e^{(N-\nu) z} \sum_{\mu=0}^{\infty} b_{\mu} z^{\mu}
$$

which implies the recursion formula in (2.23), where $b_{0}=a_{0}=1$ in view of (2.9). Note that $b_{n}>0$ for $n \in \mathbb{N}_{0}$ in the case $c_{\nu}>0(\nu=0,1, \ldots, N)$.

Example 2.8 (De Rham's function). Denoting by $\varphi(t, a)$ de Rham's function with the parameter $a \in(0,1)$, the function

$$
\varphi_{0}(t)= \begin{cases}0 & \text { for } t \leq 0 \\ \varphi(t, a) & \text { for } 0 \leq t \leq 1 \\ 1 & \text { for } t \geq 1\end{cases}
$$

belongs to $\mathcal{F}_{0}$ and it is an eigenfunction of (1.1) to the eigenvalue $\lambda_{0}=1$ and the characteristic polynomial $P(w)=a+(1-a) w$ (cf. [7]). Here we have $N=1, c_{0}=a$, $c_{1}=1-a$, where in the recursions (2.21) and (2.23) the last sum consists of one term only, namely $1-a$ and $a$, respectively, and (2.23) yields [4: Equation (3.11)]. In [4] there are also contained explicitly the first $b_{n}$ up to $n=5$. If we denote the coefficients more precisely by $a_{n}(a)$ and $b_{n}(a)$, respectively, then the both recursions imply the equation $a_{n}(1-a)=(-1)^{n} b_{n}(a)$ for $n \in \mathbb{N}_{0}$, which is nothing else but relation (4.16) for $t=0$ from [4: Proposition 4.2].

Finally, in this section we come back to Proposition 2.1 in the general case. Condition (1.4) for comparison sequences implies that there exists an integer $n_{0}$ such that $f_{n}$ is continuous so far as $n \geq n_{0}$. For a continuous function $f_{n} \in \mathcal{F}_{n}$ we introduce the generating function

$$
F_{n}(w)=\sum_{k=1}^{\infty} f_{n}(k) w^{k-1}
$$

of its values at integer arguments. In the case $n<0$ the series terminates and $F_{n}$ is a polynomial. In the case $n \geq 0$ the series converges for $|w|<1$ and we shall show that $F_{n}$ always is a rational function. For this reason we recall the Euler-Frobenius polynomials $E_{\nu}$ (cf. $\left.[10,20]\right)$ which are defined by

$$
\frac{E_{\nu}(w)}{(1-w)^{\nu+1}}=\sum_{k=1}^{\infty} k^{\nu} w^{k-1} \quad\left(\nu \in \mathbb{N}_{0}\right)
$$

and we require in Proposition 2.1 that $s \in \mathbb{N}_{0}$. 
Lemma 2.9. For $n \geq \max \left(0, n_{0}\right)$, the generating function (2.25) is a rational function which can either be written as

$$
F_{n}(w)=\sum_{k=1}^{s} f_{n}(k) w^{k-1}+w^{s} \sum_{\nu=0}^{n} \frac{b_{n-\nu}}{\nu !} \frac{E_{\nu}(w)}{(1-w)^{\nu+1}}
$$

where the coefficients $b_{\nu}$ are given by (2.8) and $E_{\nu}$ by (2.26), or in the form

$$
F_{n}(w)=\sum_{k=1}^{s} f_{n}(k) w^{k-1}+w^{s} \sum_{\nu=0}^{n} \frac{d_{\nu}}{(1-w)^{\nu+1}}
$$

where $d_{\nu}=\nabla^{\nu} p_{n}(s)$ are the backward differences with $\nabla p(s)=p(s)-p(s-1)$. Moreover, $F_{n}$ has the property

$$
\lim _{w \rightarrow 1}(1-w)^{n+1} F_{n}(w)=1 .
$$

Proof. According to continuity, equation (2.11) is even valid for $t \geq s$. Hence we find by means of the Taylor formula the equation

$$
F_{n}(w)-\sum_{k=1}^{s} f_{n}(k) w^{k-1}=\sum_{k=1}^{\infty} p_{n}(s+k) w^{s+k-1}=w^{s} \sum_{\nu=0}^{n-1} \frac{p_{n}^{(\nu)}(s)}{\nu !} \sum_{k=1}^{\infty} k^{\nu} w^{k-1}
$$

which implies representation $(2.27)$ in view of $p_{n}^{(\nu)}(s)=b_{n-\nu}($ cf. $(2.8))$.

In order to obtain the representation $(2.28)$, we interpret $p_{n}(s+t)$ as interpolation polynomial of Newton

$$
p_{n}(s+t)=\sum_{\nu=0}^{n} d_{\nu}\left(\begin{array}{c}
t+\nu-1 \\
\nu
\end{array}\right)
$$

(cf. [18: p. 94]) and we get

$$
F_{n}(w)-\sum_{k=1}^{s} f_{n}(k) w^{k-1}=\sum_{k=1}^{\infty} p_{n}(s+k) w^{s+k-1}=w^{s} \sum_{\nu=0}^{n} d_{\nu} \sum_{k=1}^{\infty}\left(\begin{array}{c}
k+\nu-1 \\
\nu
\end{array}\right) w^{k-1}
$$

Hence, (2.28) follows from the well known binomial series. The limit relation (2.29) follows both from (2.27) and (2.28) in view of $d_{n}=b_{0}=1$ and $E_{n}(1)=n$ !

Obviously, in Lemma 2.9 the rational function $F_{n}(w)$ has exactly one single pole, which lies at $w=1$ and which has the order $n+1$. Conversely, every rational function with these properties possesses a Taylor expansion, the coefficients of which are eventually polynomials of degree $n$. 


\section{Equivalent eigenfunctions}

In the following we deal with connection (1.8) between eigenfunctions $\varphi_{n}$ of (1.1) with $\lambda=2^{n}$ and $\tilde{\varphi}_{m}$ of (1.1) with $\tilde{\lambda}=2^{m}$. For equation (1.7) we use analogous assumptions and notations as for (1.1), in particular $\tilde{c}_{0} \tilde{c}_{\tilde{N}} \neq 0, \tilde{P}(1)=1$ and also

$$
\tilde{\phi}(z)=\mathcal{L}\left\{\tilde{\phi}_{-1}\right\}=\prod_{j=1}^{\infty} \tilde{P}\left(e^{-z / 2^{j}}\right)
$$

(cf. (2.18) and (2.20)).

Definition 3.1. For fixed $n, m \in \mathbb{Z}$ the eigenfunctions $\varphi_{n}$ and $\tilde{\varphi}_{m}$ are called equivalent if they satisfy an equation of the form

$$
\varphi_{n}(t)=\sum_{k=0}^{\infty} r_{k} \tilde{\varphi}_{m}(t-k) \quad(t \in \mathbb{R}),
$$

with certain constants $r_{k}$, and we write $\varphi_{n} \sim \tilde{\varphi}_{m}$. The characteristic polynomials $P$ and $\tilde{P}$ are called equivalent and we write $P \sim \tilde{P}$, if there exists a constant $\alpha \in \mathbb{Z}$ such that $\varphi_{n} \sim \tilde{\varphi}_{m}$ for

$$
m-n=\alpha .
$$

The relation $\varphi_{n} \sim \tilde{\varphi}_{m}$ is indeed an equivalence relation, i.e. it is reflexive and transitive and it is also symmetric as we shall see at once. The series in (3.2) always terminates according to $\tilde{\varphi}_{m}(t)=0$ for $t<0$. In particular, (3.2) implies the relation

$$
\varphi_{n}(t)=r_{0} \tilde{\varphi}_{m}(t) \quad(t<1)
$$

between equivalent eigenfunctions.

Since eigenfunctions never vanish identically in a right neighbourhood of $t=0$, we have

Lemma 3.2. A necessary condition for eigenfunctions $\varphi_{n}$ and $\tilde{\varphi}_{m}$ to be equivalent is that $r_{0} \neq 0$ in $(3.2)$.

According to $r_{0} \neq 0$ equation (3.2) can recursively be inverted for $t<\ell \quad(\ell \in \mathbb{N})$, which shows the symmetry of the equivalence.

By differentiation or integration of (3.2), we see that $\varphi_{n} \sim \tilde{\varphi}_{m}$ implies $\varphi_{n+k} \sim \tilde{\varphi}_{m+k}$ for arbitrary $k \in \mathbb{Z}$ (cf. (1.4)). Hence, $\varphi_{n} \sim \tilde{\varphi}_{m}$ is tantamount to $P \sim \tilde{P}$. In particular, for $P \sim \tilde{P}$ we can assume $m$ and $n$ in (3.3) so great that the eigenfunctions $\varphi_{n}$ and $\tilde{\varphi}_{m}$ are continuous so that we can introduce as in (2.25) the generating functions

$$
\Phi_{n}(w)=\sum_{k=1}^{\infty} \varphi_{n}(k) w^{k-1}, \quad \tilde{\Phi}_{m}(w)=\sum_{k=1}^{\infty} \tilde{\varphi}_{m}(k) w^{k-1}
$$

for $|w|<1$. Moreover, we introduce the generating function

$$
R(w)=\sum_{k=0}^{\infty} r_{k} w^{k}
$$

of the coefficients in (3.2), at first as a formal power series. 
Proposition 3.3. If the continuous eigenfunctions $\varphi_{n}$ and $\tilde{\varphi}_{m}$ are equivalent, then the generating function (3.6) is rational and it is representable as

$$
R(w)=\frac{\Phi_{n}(w)}{\tilde{\Phi}_{m}(w)} .
$$

Moreover, we have the limit relation

$$
\lim _{w \rightarrow 1} \frac{R(w)}{(1-w)^{\alpha}}=1
$$

with $\alpha$ from (3.3).

Proof. For $t=\ell \in \mathbb{N}_{0}$ equation (3.2) reduces to

$$
\varphi_{n}(\ell)=\sum_{k=0}^{\ell-1} r_{k} \tilde{\varphi}_{m}(\ell-k) .
$$

Multiplication by $w^{\ell-1}$ and summation over $\ell$ yields (3.7) in view of (3.6) and (3.5). Applying Lemma 2.9 both to $\Phi_{n}$ and $\tilde{\Phi}_{m}$, we first see that $R$ is rational and second that (3.8) is valid in view of (2.29) and (3.3)

\section{Corollary 3.4.}

1. The rationality of $R$ implies that the series (3.6) always converges for sufficiently small $|w|$.

2. The function $R$ in (3.7) is independent of the indices $m$ and $n$ so long as they are great enough and (3.3) is valid.

There exists a second representation of $R$.

\section{Proposition 3.5.}

1. If the eigenfunctions $\varphi_{n}$ and $\tilde{\varphi}_{m}$ are equivalent, then for $w=e^{-z} \neq 0$ the generating function (3.6) of the coefficients in (3.2) is representable in the form

$$
R\left(e^{-z}\right)=\frac{z^{\alpha} \phi(z)}{\tilde{\phi}(z)}
$$

with (2.18), (3.1) and (3.3).

2. Conversely, if the function $R(w)$, given for $w=e^{-z} \neq 0$ by (3.9), possesses in a neighbourhood of $w=0$ an expansion of the form (3.6), then $\varphi_{n} \sim \tilde{\varphi}_{m}$ so far as (3.3) is satisfied.

Proof. With notation (3.6), equation (3.2) yields by Laplace transform

$$
\mathcal{L}\left\{\varphi_{n}\right\}=R\left(e^{-z}\right) \mathcal{L}\left\{\tilde{\varphi}_{m}\right\} .
$$

According to (1.5), the entire functions (2.18) and (3.1) can be written as

$$
\phi(z)=z^{n+1} \mathcal{L}\left\{\varphi_{n}\right\}, \quad \tilde{\phi}(z)=z^{m+1} \mathcal{L}\left\{\tilde{\varphi}_{m}\right\}
$$

so that equation (3.10) turns over into (3.9) with (3.3). Conversely, if the function $R$, given by (3.9) for $w=e^{-z} \neq 0$, has a power series expansion of the form (3.6), then the foregoing steps can be inverted and (3.10) implies (3.2) 
Since expansion (3.6) in the neighbourhood of $w=0$ is only possible if function (3.9) for $w=e^{-z} \neq 0$ has a continuous continuation at $w=0$ with $R(0)=r_{0}$ and since $\phi(0)=\tilde{\phi}(0)=1$, we obtain from (3.9) and Lemma 3.2 the

Corollary 3.6. If the eigenfunctions $\varphi_{n}$ and $\tilde{\varphi}_{m}$ are equivalent, then with $r_{0}$ from (3.4) and $\alpha$ from (3.3) we have the limit relations

$$
\lim _{z \rightarrow+\infty} \frac{z^{\alpha} \phi(z)}{\tilde{\phi}(z)}=r_{0}
$$

uniformly in $\operatorname{Im} z$ and

$$
\lim _{z \rightarrow 0} \frac{R\left(e^{-z}\right)}{z^{\alpha}}=1,
$$

where the last limit is equivalent to (3.8). For the corresponding characteristic polynomials $P \sim \tilde{P}$ the constant $\alpha$ from (3.3) is uniquely determined by (3.12) and $r_{0} \neq 0$.

Example 3.7 (Splines). In the case $\tilde{P}(w)=\frac{1}{2}(1+w)$ and $P(w)=\left(\frac{1+w}{2}\right)^{\ell}$ with $\ell \in \mathbb{N}_{0}$, where the eigenfunctions are splines $[17,20]$ we find for the corresponding functions (2.20)

$$
\tilde{\phi}(z)=\prod_{j=1}^{\infty} \frac{1+e^{-z / 2^{j}}}{2}=\frac{1-e^{-z}}{z}, \quad \phi(z)=\left(\frac{1-e^{-z}}{z}\right)^{\ell},
$$

and (3.9) implies $R\left(e^{-z}\right)=z^{\alpha+1-\ell}\left(1-e^{-z}\right)^{\ell-1}$. For $w=e^{-z}$ the function $R$ possesses expansion (3.6) if and only if $\alpha=\ell-1$ so that $R(w)=(1-w)^{\alpha}$ with $R(0)=r_{0}=1$. Hence according to Proposition 3.5/2 we have equivalence relation (3.2). Choosing $m=-1$ and therefore $n=m-\alpha=-\ell$, equation (3.2) turns over into the elementary representation

$$
\varphi_{-\ell}(t)=\sum_{k=0}^{\ell-1}\left(\begin{array}{c}
\ell-1 \\
k
\end{array}\right)(-1)^{k} \chi_{[0,1)}(t-k) \quad(t \in \mathbb{R}),
$$

where $\tilde{\varphi}_{-1}=\chi_{[0,1)}$ is the characteristic function of $[0,1)$.

The different possibilities (3.7) and (3.9) to represent $R$ cause a new relation, which can be considered as a certain generalization of (3.2):

Proposition 3.8. Continuous equivalent eigenfunctions $\varphi_{n}$ and $\tilde{\varphi}_{m}$ always satisfy

$$
\sum_{k=0}^{\infty} \varphi_{n}(k) \tilde{\varphi}_{m}(t-k)=\sum_{k=0}^{\infty} \tilde{\varphi}_{m}(k) \varphi_{n}(t-k) \quad(t \in \mathbb{R}) .
$$

Proof. From (3.7) and (3.9) with $\alpha=m-n$ it follows

$$
z^{n} \Phi_{n}\left(e^{-z}\right) \tilde{\phi}(z)=z^{m} \tilde{\Phi}_{m}\left(e^{-z}\right) \phi(z) .
$$

Dividing this equation by $z^{m+n+1}$, considering (3.5) as well as (3.11) and applying the inverse Laplace transform, we get relation (3.14)

For $t \in \mathbb{N}$ equation (3.14) reduces to the commutative law of the Cauchy product. 


\section{The basic functional equation}

The sufficient condition of Proposition 3.5 has the disadvantage that it is impossible to check it if the functions $\phi$ and $\tilde{\phi}$ are not known explicitly. For this reason, we look for a representation of $R$ by means of the given characteristic polynomials.

\section{Proposition 4.1.}

1. The function $R$, defined for $w=e^{-z} \neq 0$ by (3.9), satisfies the homogeneous equation

$$
2^{\alpha} P(w) R(w)=\tilde{P}(w) R\left(w^{2}\right) .
$$

2. Conversely, every solution of (4.1) with the property

$$
\lim _{z \rightarrow 0} \frac{R\left(e^{-z}\right)}{z^{\alpha}}=R_{1}
$$

has the representation

$$
R\left(e^{-z}\right)=R_{1} \frac{z^{\alpha} \phi(z)}{\tilde{\phi}(z)} .
$$

Proof. 1. We multiply (3.9) by the same representation, only with $2 z$ instead of $z$, so that we obtain

$$
(2 z)^{\alpha} \phi(2 z) \tilde{\phi}(z) R\left(e^{-z}\right)=z^{\alpha} \phi(z) \tilde{\phi}(2 z) R\left(e^{-2 z}\right) .
$$

By means of (2.19), the last equation turns over into

$$
2^{\alpha} P\left(e^{-z}\right) R\left(e^{-z}\right)=\tilde{P}\left(e^{-z}\right) R\left(e^{-2 z}\right)
$$

and for $w=e^{-z}$ into (4.1).

2. Conversely, if we replace $z$ by $\frac{z}{2}$ and write (4.4) in the form

$$
R\left(e^{-z}\right)=2^{\alpha} R\left(e^{-z / 2}\right) \frac{P\left(e^{-z / 2}\right)}{\tilde{P}\left(e^{-z / 2}\right)},
$$

we obtain by iteration

$$
R\left(e^{-z}\right)=2^{\alpha \ell} R\left(e^{-z / 2^{\ell}}\right) \prod_{j=1}^{\ell} \frac{P\left(e^{-z / 2^{j}}\right)}{\tilde{P}\left(e^{-z / 2^{j}}\right)}
$$

for arbitrary $\ell \in \mathbb{N}$. In view of (4.2), i.e.

$$
\lim _{\ell \rightarrow \infty}\left(\frac{2^{\ell}}{z}\right)^{\alpha} R\left(e^{-z / 2^{\ell}}\right)=R_{1}
$$

(2.20) and (3.1), this implies assertion (4.3) 
We call (4.1) the basic functional equation, which contains besides $R$ and the complex variable $w$ only given data. Temporarily we permit that the parameter $\alpha$ in (4.1) is an arbitrary complex number. We look for solutions $R$ with expansion (3.6) and $R(0)=r_{0} \neq 0$ (cf. Lemma 3.2) and remember that our characteristic polynomials never vanish for $w=0$.

\section{Proposition 4.2.}

1. The basic functional equation (4.1) has a solution $R$ which is continuous at $w=0$ with $R(0) \neq 0$ if and only if the condition

$$
\tilde{P}(0)=2^{\alpha} P(0)
$$

is satisfied. In the case (4.5) this solution reads

$$
R(w)=R(0) \prod_{j=0}^{\infty} \frac{\tilde{P}\left(w^{2^{j}}\right)}{2^{\alpha} P\left(w^{2^{j}}\right)},
$$

and it is even holomorphic at least for $|w|<\max (1, \varrho)$, where $\varrho$ is the smallest number with $P(w) \neq 0$ for $|w|<\varrho$. Given $R(0)$, there is no further solution of (4.1) which is continuous at $w=0$.

2. The coefficients of the power series (3.6) for solution (4.6) are uniquely determined by $r_{0}=R(0)$ and the system

$$
2^{\alpha} \sum_{\nu+k=\ell} r_{k} c_{\nu}=\sum_{\nu+2 k=\ell} r_{k} \tilde{c}_{\nu} \quad(\ell \in \mathbb{N})
$$

where $c_{\nu}=0$ for $\nu>N$ and $\tilde{c}_{\nu}=0$ for $\nu>\tilde{N}$.

Proof. 1. If equation (4.1) has a solution $R$ which is continuous at $w=0$ with $R(0) \neq 0$, then equation (4.1) implies (4.5) for $w \rightarrow 0$. Moreover, if we write (4.1) in the form

$$
R(w)=\frac{\tilde{P}(w)}{2^{\alpha} P(w)} R\left(w^{2}\right)
$$

we obtain by iteration

$$
R(w)=R\left(w^{2^{\ell}}\right) \prod_{j=0}^{\ell-1} \frac{\tilde{P}\left(w^{2^{j}}\right)}{2^{\alpha} P\left(w^{2^{j}}\right)}
$$

for arbitrary $\ell \in \mathbb{N}$. For $\ell \rightarrow \infty$ this shows that a solution $R$ of (4.1), which is continuous at $w=0$, must have representation (4.6).

Conversely, if (4.5) is satisfied, then the product in (4.6) is uniformly convergent in every compact subset of $|w|<\max (1, \varrho)$. Hence it represents a holomorphic function there, and it can immediately be checked that it satisfies equation (4.1).

2. Substituting series (3.6) into (4.1) it can easily be seen by comparison of coefficients that system (4.7) is equivalent to equation (4.1). The coefficients $r_{k}$ are uniquely determined by (4.7) up to a constant factor, which is fixed by $r_{0}=R(0)$ 
If we choose $R(0)=r_{0}=1$, then from (4.7) we obtain for the next two coefficients

$$
\begin{aligned}
& r_{1}=\frac{2^{-\alpha} \tilde{c}_{1}-c_{1}}{c_{0}} \\
& r_{2}=\frac{2^{-\alpha} \tilde{c}_{2}-c_{2}}{c_{0}}+\frac{\left(2^{-\alpha} \tilde{c}_{1}-c_{1}\right)\left(2^{-\alpha} \tilde{c}_{0}-c_{1}\right)}{c_{0}^{2}}
\end{aligned}
$$

A consequence of Propositions 4.1 and 4.2 is the following

Corollary 4.3. The solutions (4.3) and (4.6) of (4.1) coincide for suitable constants $R_{1}$ and $R(0)$, if either (4.3) with $w=e^{-z}$ possesses a continuous continuation at $w=0$ with $R(0) \neq 0$ or if (4.6) has property (4.2) with $R_{1} \neq 0$. In these cases, the limit (3.12) exists with $r_{0} \neq 0$, the constants are connected by $R(0)=r_{0} R_{1}$, and $R$ has expansion (3.6).

In the following we fix the branch of the complex power function $w^{\alpha}$ by $1^{\alpha}=1$.

Proposition 4.4. Let $R$ be a non-trivial solution of the basic equation (4.1).

1. The general solution of (4.1) reads

$$
U\left(\frac{\ln (-\ln w)}{\ln 2}\right) R(w)
$$

where $U(\cdot)$ is an arbitrary 1-periodic function.

2. Let $R$ moreover have at least one of the representations

$$
R(w)=w^{\alpha_{0}} R_{0}(w) \quad \text { or } \quad R(w)=(1-w)^{\alpha_{1}} R_{1}(w)
$$

where $R_{j}$ are continuous functions at $w=j, R_{j}(j) \neq 0$ and $\alpha_{j} \in \mathbb{C}$ for $j \in\{0,1\}$. Then in the first case of (4.10) $\alpha_{0}=0$, and in the second case $\alpha_{1} \equiv \alpha \bmod \frac{2 \pi i}{\ln 2}$.

Proof. 1. The quotient $Q(w)$ of two solutions of (4.1) satisfies $Q(w)=Q\left(w^{2}\right)$, and this equation has the general solution

$$
Q(w)=U\left(\frac{\ln (-\ln w)}{\ln 2}\right)
$$

with $U(\cdot)$ as above, so that the first assertion is proved.

2. The replacement of (4.10) into (4.1) yields

$$
2^{\alpha} P(w) R_{j}(w)=(j+w)^{\alpha_{j}} \tilde{P}(w) R_{j}\left(w^{2}\right)
$$

$j \in\{0,1\}$, and for $w \rightarrow j$ we obtain $\alpha_{0}=0$ (and the already known condition (4.5)) in the first case of (4.10), and $\alpha_{1} \equiv \alpha \bmod \frac{2 \pi i}{\ln 2}$ in the second case, since $P(1)=\tilde{P}(1)=1$ 


\section{Remark 4.5.}

1. The solutions of (4.1) with the first property in (4.10) are the solutions (4.6), and the solutions with the second property are the solutions (4.3) with $R_{1}=R_{1}(0)$. There are no further solutions with (4.10), since $U$ in (4.9) must be constant in these cases.

2. For a non-trivial rational solution $R$ of (4.1) conditions (4.10) are always satisfied. Hence, the existence of such a solution implies both $R(0) \neq 0$ and $\alpha_{1}=\alpha$ in (4.10) since, without loss of generality, we can assume here that $\alpha \in \mathbb{R}$.

For the next considerations we also temporarily abandon that $\lambda$ in (1.1) and $\tilde{\lambda}$ in (1.7) are eigenvalues, and we only require that the corresponding solutions $\varphi$ and $\tilde{\varphi}$ are generalized functions satisfying the boundary condition (i). The following proposition is a generalization of [4: Proposition 5.1].

Proposition 4.6. If $\tilde{\varphi}$ is an arbitrary solution of (1.7) satisfying $(i)$, and if (4.5) is satisfied with

$$
2^{\alpha}=\frac{\tilde{\lambda}}{\lambda}
$$

then the generalized function

$$
\varphi(t)=\sum_{k=0}^{\infty} r_{k} \tilde{\varphi}(t-k) \quad(t \in \mathbb{R})
$$

is such a solution of (1.1), where $r_{k}$ are the coefficients in (3.6) of the solution (4.6) of (4.1) with an arbitrary $r_{0}=R(0)$.

Proof. Using (4.7) and (4.11), a straightforward calculation shows indeed:

$$
\begin{aligned}
\lambda \varphi\left(\frac{t}{2}\right) & =\frac{\tilde{\lambda}}{2^{\alpha}} \sum_{k=0}^{\infty} r_{k} \tilde{\varphi}\left(\frac{t-2 k}{2}\right) \\
& =\frac{1}{2^{\alpha}} \sum_{k=0}^{\infty} r_{k} \sum_{\nu=0}^{\tilde{N}} \tilde{c}_{\nu} \tilde{\varphi}(t-2 k-\nu) \\
& =\frac{1}{2^{\alpha}} \sum_{\ell=0}^{\infty} \sum_{\nu+2 k=\ell} r_{k} \tilde{c}_{\nu} \tilde{\varphi}(t-\ell) \\
& =\sum_{\ell=0}^{\infty} \sum_{\nu+k=\ell} c_{\nu} r_{k} \tilde{\varphi}(t-\ell) \\
& =\sum_{\nu=0}^{N} c_{\nu} \sum_{k=0}^{\infty} r_{k} \tilde{\varphi}(t-\nu-k) \\
& =\sum_{\nu=0}^{N} c_{\nu} \varphi(t-\nu)
\end{aligned}
$$

so that the assertion is proved 
We give three applications of this proposition.

Lemma 4.7. Let be $r_{0} \neq 0$. Then relation (4.12) can always be inverted by

$$
\tilde{\varphi}(t)=\sum_{k=0}^{\infty} s_{k} \varphi(t-k),
$$

where the coefficients $s_{k}$ are those in

$$
S(w)=\sum_{k=0}^{\infty} s_{k} w^{k}
$$

of the function $S=\frac{1}{R}$ with $R$ from (4.6).

Proof. Introducing the function $S=\frac{1}{R}$ with (4.6) and $R(0)=r_{0} \neq 0$, equation (4.1) can also be written as $2^{-\alpha} \tilde{P}(w) S(w)=P(w) S\left(w^{2}\right)$. The function $S$ is holomorphic for $|w|<\max \{1, \tilde{\varrho}\}$, where $\tilde{\varrho}$ is the smallest number with $\tilde{P}(w) \neq 0$ for $|w|<\tilde{\varrho}$ and $S(0)=\frac{1}{R(0)} \neq 0$. Hence, applying Proposition 4.6 with power series (4.14) instead of (3.6), we obtain relation (4.13)

Next, we consider the constant characteristic polynomial $\tilde{P}(w)=1$, where $(1.7)$ reduces to

$$
\tilde{\lambda} \tilde{\varphi}\left(\frac{t}{2}\right)=\tilde{\varphi}(t) .
$$

For $t>0$ the solutions of this equation have the form

$$
\tilde{\varphi}(t)=t^{\gamma} V\left(\frac{\ln t}{\ln 2}\right)
$$

where $\gamma=\frac{\ln \tilde{\lambda}}{\ln 2}$ and where $V(\cdot)$ is a 1-periodic generalized function. By application of Proposition 4.6 it follows

Corollary 4.8. Choosing $\tilde{\lambda}=\frac{\lambda}{c_{0}}, 2^{\alpha}=\frac{1}{c_{0}}$ and $r_{0}=1$, every solution of (4.15) with (i) generates a generalized function (4.12) which is a solution of (1.1).

Since $\varphi(t)=\tilde{\varphi}(t)$ for $t<1$, we can interpret formula (4.12) with $r_{0}=1$ as the explicit representation of $\varphi$ by means of its restriction to $t<1$. With other words, this representation yields uniquely the extension of the restriction to the case of a solution for arbitrary real $t$. A third consequence of Proposition 4.6 reads:

Corollary 4.9. Let (4.11) and

$$
\frac{\lambda}{c_{0}}=\frac{\tilde{\lambda}}{\tilde{c}_{0}}
$$

be satisfied, and let $\varphi$ and $\tilde{\varphi}$ be solutions of (1.1) and (1.7), respectively, satisfying (i) and

$$
\varphi(t)=r_{0} \tilde{\varphi}(t) \quad(t<1) .
$$

Then this equation can be extended by means of (4.12) with $r_{k}$ from (4.7).

In the following we come back to $\alpha \in \mathbb{Z}$ and also to eigenfunctions $\varphi_{n}$ of (1.1) and $\tilde{\varphi}_{m}$ of (1.7) with $\lambda=2^{n}$ and $\tilde{\lambda}=2^{m}$. Then (3.3) and (4.11) are equivalent as well as (4.5) and (4.16), and (4.13) is the explicit inversion of (3.2). Moreover, application of Corollary 4.9 shows that equation (3.4) can always be extended to (3.2), which means that Definition 3.1 can be replaced by the simpler 
Definition 4.10. The eigenfunctions $\varphi_{n}$ and $\tilde{\varphi}_{m}$ are called equivalent, i.e. $\varphi_{n} \sim$ $\tilde{\varphi}_{m}$, if they satisfy equation (3.4) with a certain constant $r_{0}$.

Using this definition, the symmetry of the equivalence is trivial in view of Lemma 3.2 .

\section{The main equivalence theorem}

After these preparations we state the main result concerning equivalent eigenfunctions and equivalent characteristic polynomials, respectively, and the basic functional equation.

Theorem 5.1. With notation (3.3) the following assertions are equivalent:

(a) $\varphi_{n} \sim \tilde{\varphi}_{m}$, i.e. $P \sim \tilde{P}$.

(b) The basic functional equation (4.1) has a non-trivial rational solution $R$.

(c) There exists limit (3.12) with $r_{0} \neq 0$ uniformly in $\operatorname{Im} z$.

Proof. $(\mathrm{a}) \Rightarrow(\mathrm{b})$ : According to Proposition 3.3 the generating function $R$ of the coefficients in $(3.2)$ is rational with $R(0) \neq 0$. Moreover, owing to Proposition 3.5, for $w=e^{-z} \neq 0, R$ is representable in form (3.9), and in view of Proposition 4.1 we see that $R$ is a solution of (4.1).

(b) $\Rightarrow(\mathrm{c})$ : If $R$ is a non-trivial rational solution of (4.1), then according to Remark $4.5 / 2$ this solution has the structure

$$
R(w)=(1-w)^{\alpha} R_{1}(w)
$$

with $R_{1}(1) \neq 0$. From $(5.1)$ we obtain

$$
\lim _{w \rightarrow 1} \frac{R(w)}{(1-w)^{\alpha}}=R_{1}(1)
$$

i.e. (4.2) with $R_{1}=R_{1}(1)$. Hence, Proposition 4.1/2 yields representation (4.3), and the continuity of $R$ at $w=0$ implies the uniform existence of limit (3.12) with

$$
r_{0}=\frac{R(0)}{R_{1}(1)} \neq 0
$$

$(\mathrm{c}) \Rightarrow(\mathrm{a})$ : Assume that there exists limit (3.12) with $r_{0} \neq 0$ uniformly in $\operatorname{Im} z$. From Proposition 4.1/1 we know that the function $R$ from (3.9) with $e^{-z}=w$ is a solution of (4.1). According to (3.12) it is continuous at $w=0$ with $R(0)=r_{0} \neq 0$, and Proposition 4.2/1 implies that it even possesses power series (3.6). Hence, Proposition $3.5 / 2$ yields $\varphi_{n} \sim \tilde{\varphi}_{m}$ with $m-n=\alpha$

According to this theorem we only have to look for non-trivial rational solutions $R$ of the basic functional equation (4.1). For such solutions both conditions of (4.10) are satisfied so that both statements of Proposition 4.4/2 come true, but we also have the inversion: 
Proposition 5.2. If a solution $R$ of (4.1) with $\alpha \in \mathbb{Z}$ satisfies both conditions (4.10), then it is a rational function.

Proof. Let $R$ be a (non-trivial) solution of (4.1) with $\alpha \in \mathbb{Z}$ satisfying both conditions (4.10). In the proof $(\mathrm{b}) \Rightarrow$ (c) of Theorem 5.1 we only have used these properties of $R$ and not its rationality. Hence, it follows in the same way (3.12) with $r_{0} \neq 0$ uniformly in $\operatorname{Im} z$, and from Theorem 5.1 that $R$ is a rational function

In addition to Corollary 4.3 we now can say in the case $\alpha \in \mathbb{Z}$ that solutions (4.3) and (4.6) of (4.1) coincide if and only if these solutions are rational functions with $R(0)=r_{0} R_{1}$, where $r_{0}$ is the limit (3.12). The rational solutions are determined up to a constant factor (cf. Remark 4.5), but we need such a solution with a fixed factor.

Definition 5.3. A rational solution of the basic functional equation (4.1) is called the canonical solution, if it has property (3.8).

With other words, the canonical solution is a rational solution with the normalization $R_{1}(1)=1$ of the function $R_{1}(w)$ in (5.1). In view of (5.2) this condition is equivalent to $R(0)=r_{0}$, where $r_{0}$ is given by (3.12), however, condition (3.8) is easiler to handle than condition (3.12) in order to determine the correct factor of a canonical solution.

For applications Theorem 5.1 must be completed by

Corollary 5.4. In (3.2) the coefficients $r_{k}$ of equivalent eigenfunctions $\varphi_{n}$ and $\tilde{\varphi}_{m}$ are the coefficients in (3.6) of the canonical solution $R$ of the basic functional equation (4.1). These coefficients are determined by means of (4.7) and $R_{1}(1)=1$ in (5.1).

\section{Minimal characteristic polynomials}

In this section we are going to minimize the degree $N$ of (1.2) in the class of equivalent characteristic polynomials in order to get a survey on the entire equivalence class.

Definition 6.1. A characteristic polynomial $\tilde{P}$ is called minimal if $P \sim \tilde{P}$ implies that $N \geq \tilde{N}$.

Obviously, in view of the degree condition, every equivalence class of characteristic polynomials possesses at least one minimal polynomial. In the following we want to characterize both equivalent and minimal characteristic polynomials, and we shall show the uniqueness of minimal characteristic polynomials. For this reason, we first write the basic functional equation (4.1) in the form

$$
P(w) R_{1}(w)=\left(\frac{1+w}{2}\right)^{\alpha} \tilde{P}(w) R_{1}\left(w^{2}\right)
$$

where $R_{1}(w)$ is the rational function of (5.1) with $R_{1}(1)=1$ and $\alpha \in \mathbb{Z}$. 
Proposition 6.2. Let $\tilde{P}$ be a minimal characteristic polynomial. Then $P \sim \tilde{P}$ if and only if $P$ has the form

$$
P(w)=\left(\frac{1+w}{2}\right)^{\alpha} \frac{p\left(w^{2}\right)}{p(w)} \tilde{P}(w)
$$

where $p$ is a polynomial with $p(0) p(1) \neq 0$, and $\alpha \in \mathbb{N}_{0}$.

Proof. If $P \sim \tilde{P}$, then equation (6.1) has a non-trivial rational solution $R_{1}=\frac{p}{q}$ in reduced representation, i.e. the polynomials $p$ and $q$ have no common zeros, and the values $p(0), p(1), q(0)$ and $q(1)$ are different from zero according to $R_{1}(0) \neq 0$ and $R_{1}(1) \neq 0$. Moreover, the function $P_{0}$ defined by

$$
P_{0}(w)=\left(\frac{2}{1+w}\right)^{\alpha} \frac{p(w)}{p\left(w^{2}\right)} P(w)=\frac{q(w)}{q\left(w^{2}\right)} \tilde{P}(w)
$$

is equal to a polynomial with $P_{0}(1)=1$, and we get

$$
P(w)=\left(\frac{1+w}{2}\right)^{\alpha} \frac{p\left(w^{2}\right)}{p(w)} P_{0}(w), \quad \tilde{P}(w)=\frac{q\left(w^{2}\right)}{q(w)} P_{0}(w) .
$$

Therefore according to (6.1) we have $P_{0} \sim P$ and $P_{0} \sim \tilde{P}$. Since the polynomial $\tilde{P}$ is minimal, the second equation of (6.3) implies that the polynomial $q$ must be a constant $\neq 0$, i.e. $\tilde{P}(w)=P_{0}(w)$, and the first equation of (6.3) implies (6.2). If $\alpha$ would be negative, then $(1+w)^{-\alpha}$ must be a factor of $\tilde{P}$, because it cannot be a factor of $p\left(w^{2}\right)$ in view of $p(1) \neq 0$. Hence,

$$
\tilde{P}(w)=\frac{2^{\alpha}}{(1+w)^{\alpha}} P_{1}(w)=2^{\alpha} \frac{(1-w)^{\alpha}}{\left(1-w^{2}\right)^{\alpha}} P_{1}(w)
$$

with a certain polynomial $P_{1}$, so that $P_{1} \sim \tilde{P}$ according to (4.1) with $R(w)=(1-w)^{\alpha}$, and $\tilde{P}$ would not be minimal. This is a contradiction to our assumption, and we have proved $\alpha \geq 0$. Conversely, if (6.2) is satisfied, then also (6.1) with $R_{1}=p$, and we have $P \sim \tilde{P}$

From the proof we immediately see the validity of the following statements.

\section{Corollary 6.3.}

1. If $P \sim \tilde{P}$, then there are polynomials $p$ and $q$ with $p(1) q(1) \neq 0$, and a characteristic polynomial $P_{0}$ such that the two equations (6.3) hold with $\alpha$ from (4.1).

2. If $P \sim \tilde{P}$ and $\tilde{P}$ is minimal, then the canonical solution of (4.1) reads $R(w)=$ $(1-w)^{\alpha} p(w)$ with $p$ from $(6.2)$ normalized by $p(1)=1$.

3. A necessary condition for $\tilde{P}$ to be a minimal characteristic polynomial is that $\tilde{P}(-1) \neq 0$.

Remark 6.4. Representation (6.2) means that the characteristic polynomial $P$ is equivalent to the minimal polynomial $\tilde{P}$ if and only if there exists a polynomial $Q(w)=(1-w)^{\alpha} p(w)$ with $Q(0) \neq 0$ so that

$$
P(w)=\frac{1}{2^{\alpha}} \frac{Q\left(w^{2}\right)}{Q(w)} \tilde{P}(w),
$$


where $\alpha \in \mathbb{N}_{0}$ is the order of the zero 1 of $Q$. According to Corollary $6.3 / 2$ we can choose $Q=R$. Note that $p$ in (6.2) can also have -1 as a zero, e.g. in the example $P=\frac{1}{2}\left(1+w^{2}\right)$ and $\tilde{P}=1$, where $\alpha=1, p(w)=1+w$ and $Q(w)=1-w^{2}$. However, the order $\beta$ of the zero -1 of $p$ cannot exceed $\alpha$ so that $\alpha=0$ implies $p(-1) \neq 0$.

Proposition 6.5. Every class of equivalent characteristic polynomials contains exactly one minimal polynomial.

Proof. If $P \sim \tilde{P}$ and if both characteristic polynomials are minimal, then in view of Proposition 6.2 and Remark 6.4 there are polynomials $Q_{j} \quad(j=1,2)$ with $Q_{j}(0) \neq 0$, and exponents $\alpha_{j} \in \mathbb{N}_{0}$, so that we have the equations

$$
P(w)=\frac{1}{2^{\alpha_{1}}} \frac{Q_{1}\left(w^{2}\right)}{Q_{1}(w)} \tilde{P}(w), \quad \tilde{P}(w)=\frac{1}{2^{\alpha_{2}}} \frac{Q_{2}\left(w^{2}\right)}{Q_{2}(w)} P(w)
$$

which immediately imply that $Q_{1}$ and $Q_{2}$ are constant. According to $\alpha_{j} \geq 0$ it follows $\alpha_{j}=0$ and therefore $P=\tilde{P}$

Remark 6.6. In particular, Proposition 6.5 and Remark 6.4 answer the question concerning the equivalence class with the minimal polynomial $\tilde{P}=1$. Namely, (6.4) shows that the characteristic polynomial $P$ is equivalent to the constant polynomial $\tilde{P}(w)=1$ if and only if $P$ is of the form

$$
P(w)=\frac{1}{2^{\alpha}} \frac{Q\left(w^{2}\right)}{Q(w)}
$$

with a polynomial $Q$ having the zero 1 of order $\alpha$ (cf. [5]). Note that $Q(w)$ divides $Q\left(w^{2}\right)$ if and only if the zero set $\{w \in \mathbb{C}: Q(w)=0\}$ is closed under the mapping $f_{0}: w \mapsto w^{2}$ (cf. [17: Lemma 2.3]). In particular, (6.5) implies that all zeros of $P$ and $Q$ lie on the unit circle.

In the following we need the concept of cyclic numbers under a mapping $f$, where $f$ can be one of the two mappings $f_{i}: w \mapsto(-1)^{i} w^{2} \quad(i \in\{0,1\})$.

Definition 6.7. The set of non-vanishing pairwise distinct complex numbers $\zeta_{1}, \zeta_{2}$, $\ldots, \zeta_{k}$ is called a cycle of $f$ with the length $k$ if $f\left(\zeta_{j}\right)=\zeta_{j+1}$ for $j=1,2, \ldots, k-1$ and $f\left(\zeta_{k}\right)=\zeta_{1}$. The numbers $\zeta_{1}, \ldots, \zeta_{\ell}$ are called cyclic under $f$ if each $\zeta_{j}$ belongs to a cycle of $f$ (cf. [21]).

Clearly, if the numbers $\zeta_{j}(j=1, \ldots, \ell)$ are cyclic under $f$, then the set of these numbers is one single cycle or the union of pairwise distinct cycles of $f$. Moreover, if the numbers $\zeta_{j}(j=1, \ldots, \ell)$ are cyclic under $f_{0}$, then the numbers $-\zeta_{j}$ are cyclic under $f_{1}$ and conversely. In the case that the set of numbers $\zeta_{j}$ is a cycle of $f_{i}$ with the length $k$, we have $\zeta^{2^{k}}=(-1)^{i} \zeta$ and consequently in view of $\zeta_{j} \neq 0$ that $\zeta^{2^{k}-1}=(-1)^{i}$ for each $\zeta=\zeta_{j}(j=1,2, \ldots, k)$, i.e. all cyclic numbers are roots of unity. The simplest cycle of $f_{i}$ is $\zeta_{1}=(-1)^{i} \quad(i=0,1)$ with the length $k=1$. A set of numbers which are cyclic under $f_{0}$ is also closed under $w \rightarrow w^{2}$, but not conversely, since there can appear preperiods (cf. [6]). 
Lemma 6.8. If $p$ and $q$ are polynomials with $q(0) \neq 0$ and

$$
p(w)=\frac{q\left(w^{2}\right)}{q(w)}
$$

and if $p$ has no symmetric zeros, then the zeros of $q$ are cyclic under $f_{0}$ and the zeros of $p$ are cyclic under $f_{1}$.

Proof. Let $\zeta_{1}, \ldots, \zeta_{\ell}$ be the zeros of $q$, so that

$$
p(w)=\frac{\left(w^{2}-\zeta_{1}\right) \cdots\left(w^{2}-\zeta_{\ell}\right)}{\left(w-\zeta_{1}\right) \cdots\left(w-\zeta_{\ell}\right)} .
$$

Since $p$ has no symmetric zeros, each of the factors $\left(w^{2}-\zeta_{i}\right)$ must be divisible by a certain term $\left(w-\zeta_{j}\right)$. Consequently, for each $i \in\{1,2, \ldots, \ell\}$ there is a certain $j \in\{1,2, \ldots, \ell\}$ so that $\zeta_{i}=\zeta_{j}^{2}$, and $p$ has the zeros $\xi_{j}=-\zeta_{j}$. Since numerator and denominator of (6.6) have the same number of factors it follows (if necessary after renumbering) that the zeros of $q$ are cyclic under $f_{0}$ and the zeros $\xi_{j}=-\zeta_{j}$ of $p$ are cyclic under $f_{1}$

The following result is a generalization of [10: Theorem 5.11].

Proposition 6.9. The characteristic polynomial $\tilde{P}$ is minimal if and only if the following conditions are satisfied:

(a) $\tilde{P}$ has no symmetric zeros.

(b) $\tilde{P}$ has no cyclic zeros under $f_{1}$.

Proof. 1. We assume that $\tilde{P}$ is minimal and at least one of the conditions (a) or (b) is not satisfied. According to Corollary $6.3 / 2$ we know that a zero of $\tilde{P}$ cannot be equal to -1 .

Case (a): If $\tilde{P}$ has the zeros $\zeta$ and $-\zeta$ with $\zeta^{2} \neq 1$, then we can define the polynomial

$$
P(w)=\frac{w-\zeta^{2}}{w^{2}-\zeta^{2}} \tilde{P}(w)
$$

with $P(1)=\tilde{P}(1)=1$, which has a smaller degree than $\tilde{P}$ and which is equivalent to $\tilde{P}$ according to $(6.1)$ with $R_{0}(w)=w-\zeta^{2}$ and $\alpha=0$. Hence $\tilde{P}$ cannot be minimal.

Case (b): If $\tilde{P}$ has the cyclic zeros $\zeta_{1}, \zeta_{2}, \ldots, \zeta_{\ell}$ under $f_{1}$ with $\zeta_{j} \neq-1$, then we can define the polynomial

$$
P(w)=\frac{1}{\left(w-\zeta_{1}\right)\left(w-\zeta_{2}\right) \cdots\left(w-\zeta_{\ell}\right)} \tilde{P}(w)
$$

with $P(1)=1$, which has a smaller degree than $\tilde{P}$ and which, in view of

$$
\prod_{j=1}^{\ell}\left(w-\zeta_{j}\right)=\prod_{j=1}^{\ell} \frac{w^{2}-\zeta_{j}^{2}}{w+\zeta_{j}}=\prod_{j=1}^{\ell} \frac{w^{2}-\zeta_{j}^{2}}{w-\zeta_{j}^{2}}=\frac{R_{1}(w)}{R_{1}\left(w^{2}\right)}
$$


is equivalent to $\tilde{P}$ according to (6.1) with $\alpha=0$ and

$$
R_{1}(w)=\frac{\left(1-\zeta_{1}^{2}\right) \cdots\left(1-\zeta_{\ell}^{2}\right)}{\left(w-\zeta_{1}^{2}\right) \cdots\left(w-\zeta_{\ell}^{2}\right)} .
$$

Hence $\tilde{P}$ cannot be minimal.

2. Now we consider the case that both conditions (a) and (b) are satisfied. Let $P$ be an arbitrary characteristic polynomial which is equivalent to $\tilde{P}$, we have to show that $N \geq \tilde{N}$. According to Corollary 6.3 we first show that the polynomial $q$ in $(6.3)$ is constant. For this reason, we assume on the contrary that $q$ has at least one zero. The polynomials $q$ and $P_{0}$ cannot have a common zero, since otherwise $q\left(w^{2}\right)$ and therefore also $\tilde{P}$ would have symmetric zeros, which contradicts (a), hence $\frac{q\left(w^{2}\right)}{q(w)}$ must be a polynomial without symmetric zeros. Now, Lemma 6.8 implies that all zeros of $q$ are cyclic under $f_{0}$ so that $\tilde{P}$ has cyclic zeros under $f_{1}$, which contradicts (b), and we have proved that $q$ is a non-vanishing constant in view of $q(1) \neq 0$. Hence, the second equation in (6.3) implies $P_{0}=\tilde{P}$, and the first equation turns over into (6.2), where $\alpha \geq 0$ in view of $\tilde{P}(-1) \neq 0$ and $p(1) \neq 0$. Hence, we obtain that $N \geq \tilde{N}$ and the proposition is proved

Remark 6.10. In [10] there was studied in detail the class of all such "scaling functions" (i.e. special eigenfunctions $\varphi_{-1} \in L^{2}(\mathbb{R})$ ) which generate the same multiresolution analysis. According to [10: Theorem 5.12] such scaling functions and also their corresponding characteristic polynomials, which are denoted there "two-scale symbols", are equivalent in the sense of our Definition 3.1.

In order to construct equivalent characteristic polynomials explicitly, we introduce the concept of a $w^{2}$-transformation.

Definition 6.11. The characteristic polynomial $P$ is called a $w^{2}$-transformation of $P_{0}$ and we write $P=T\left\{P_{0}\right\}$, if $P_{0}$ is a characteristic polynomial with $\operatorname{deg} P_{0} \geq 1$ and if there is a number $K \in \mathbb{N}$ such that $P(w)=P_{K}(w)$ is obtained iteratively from $P_{0}(w)$ in the following way: Choose an arbitrary linear factor $w-\zeta_{k}$ of $P_{k-1}(w)$ and put

$$
P_{k}(w)=\frac{w^{2}-\zeta_{k}}{w-\zeta_{k}} P_{k-1}(w)
$$

for $k=1,2, \ldots, K$.

Obviously, $\zeta_{k} \neq 1$ for all $k$. Hence, every $w^{2}$-transformation $P_{K}$ of $P_{0}$ possesses the representation

$$
P_{K}(w)=\frac{R\left(w^{2}\right)}{R(w)} P_{0}(w)
$$

with $R(w)=\prod_{k=1}^{K} \frac{w-\zeta_{k}}{1-\zeta_{k}}$ so that (6.9) can be intrepreted as (4.1) with $\alpha=0$ and we have $P_{K} \sim P_{0}$.

The single steps of a $w^{2}$-transformation can be gathered up, by applying the replacement $w \mapsto w^{2}$ not only in a linear factor, but in a polynomial factor or in the whole polynomial. In this form a $w^{2}$-transformation was already used in [5: Theorem 3.3] for the special case $P_{0}(w)=\frac{1}{2}(1+w)$.

Given a polynomial $\tilde{P}$ satisfying the conditions of Proposition 6.9, an arbitrary polynomial $P$ with $P \sim \tilde{P}$ can be constructed in the following way: 
Algorithm I. Let $\tilde{P}$ be an arbitrary minimal characteristic polynomial.

1. Multiply $\tilde{P}$ by an arbitrary polynomial $p$ with $p(1)=1$ having only cyclic zeros under $f_{1}$.

2. Apply a $w^{2}$-transformation to $p \tilde{P}$.

The result

$$
P(w)=T\{p(w) \tilde{P}(w)\}
$$

is a polynomial with $P \sim \tilde{P}$.

In order to see that the algorithm yields the wanted result, first we shall show that $p \tilde{P} \sim \tilde{P}$. Assume that $p$ has the zero -1 with the multiplicity $\alpha$ and the zeros $\zeta_{1}, \ldots, \zeta_{\ell}$ which are cyclic under $f_{1}$, where $\zeta_{j} \neq-1$ for $j=1, \ldots, \ell$. Then in view of $p(1)=1$ and (6.7) the polynomial $p$ has the representation

$$
p(w)=\left(\frac{1+w}{2}\right)^{\alpha} \frac{q\left(w^{2}\right)}{q(w)}
$$

with $q(w)=\left(w-\zeta_{1}^{2}\right) \cdots\left(w-\zeta_{\ell}^{2}\right)$, and we see that $P_{0}=p \tilde{P} \sim \tilde{P}$ by Proposition 6.2. Next we consider a single step (6.8) of a $w^{2}$-transformation. Comparing (6.8) with (6.1) for $\alpha=0$ and $q(w)=w-\zeta, \zeta \neq 1$, we see that $P_{k} \sim P_{k-1}$ for $k=1,2, \ldots, K$, so that the transitivity of " $\sim$ implies $P_{K}=T\{p \tilde{P}\} \sim \tilde{P}$.

Conversely, we can show by inverting the steps of Algorithm I that every characteristic polynomial $P$ which is equivalent to the minimal polynomial $\tilde{P}$, has form (6.10). This inversion leads to the minimal polynomial $\tilde{P}$ of a given characteristic polynomial $P$ :

Algorithm II. Let $P$ be an arbitrary characteristic polynomial.

1. Replace successively all quadratic factors by symmetric zeros, i.e. all factors $w^{2}-\zeta$ by $w-\zeta$.

2. Cancel all polynomial factors with only cyclic zeros under $f_{1}$.

The result is the minimal polynomial $\tilde{P}$, which is connected with $P$ by (6.10).

Let $P$ be an arbitrary characteristic polynomial $P$. We show representation $(6.10)$ by means of the corresponding minimal polynomial $\tilde{P}$.

1. If $P$ has a symmetric zero, i.e. $P(w)=\left(w^{2}-\zeta\right) q_{1}(w) \quad(\zeta \neq 0,1)$ with a certain polynomial $q_{1}$, then $P$ can be represented as

$$
P(w)=\frac{w^{2}-\zeta}{w-\zeta} Q_{1}(w), \quad Q_{1}(w)=(w-\zeta) q_{1}(w)
$$

so that $P \sim Q_{1}$ according to (6.1), and we have $Q_{1}(0) \neq 0$ and $Q_{1}(1)=1$ in view of $\zeta \neq 0,1$. Owing to (6.8), $P$ results as a single step of a $w^{2}$-transformation of $Q_{1}$. After finitely many analogous steps we obtain that $P=T\{Q\}$ and therefore $P \sim Q$, where the polynomial $Q$ has no symmetric zeros, and $Q(0) \neq 0, Q(1)=1$. 
2. Now, $P \sim \tilde{P}$ and $P \sim Q$ imply also $Q \sim \tilde{P}$ and, since $\tilde{P}$ is minimal, Proposition 6.2 yields the representation

$$
Q(w)=p(w) \tilde{P}(w), \quad p(w)=\left(\frac{1+w}{2}\right)^{\alpha} \frac{q\left(w^{2}\right)}{q(w)}
$$

with $\alpha \in \mathbb{N}_{0}$ where $q$ is a polynomial with $q(0) \neq 0$ and $q(1) \neq 0$ so that $p(1)=1$. Since $Q$ has no symmetric zeros, this is valid also for $p$, and by Lemma 6.8 the zeros of $q$ are cyclic under $f_{0}$, and the zeros of $p$ are cyclic under $f_{1}$, so that $P=T\{Q\}$ and (6.11) imply representation (6.10). Finally, Proposition 6.9 yields that $\tilde{P}$ in (6.10) is the minimal polynomial.

Note that the second step of Algorithm II is a generalization of the first one, if we permit such rational factorizations which again lead to polynomials as in (6.7), e.g.

$$
w^{2}-w+1=\frac{w^{4}+w^{2}+1}{w^{2}+w+1} \mapsto \frac{w^{2}+w+1}{w^{2}+w+1}=1 .
$$

\section{Applications}

In this section we give three applications of the foregoing results.

7.1 The sum of shifted eigenfunctions. Sum relation (1.9) is well known for Lebesgue-integrable eigenfunctions $\varphi_{-1}$ of $(1.1)$ (cf. $[5,19]$ ), but it can also be valid for distributional eigenfunctions.

Proposition 7.1. Let be $P(-1)=0$. Then we have

$$
\sum_{k=0}^{\infty} \varphi_{-1}(t-k)=1 \quad(N-1<t) .
$$

Proof. The characteristic polynomial $P$ can be written as $P(w)=\frac{1+w}{2} \tilde{P}(w)$, and this equation can be interpreted as basic functional equation (4.1) with $\alpha=1$ and the canonical solution $R(w)=1-w$. Hence, Corollary 5.4 with $m=0, n=-1$, Lemma 4.7 with $S=\frac{1}{1-w}$, and $\tilde{\varphi}_{0}(t)=1$ for $t>\tilde{N}=N-1$ yield (7.1)

Theorem 7.2. Relation (7.1) is valid if and only if the basic functional equation (4.1) with $\alpha=0$ and a certain characteristic polynomial $\tilde{P}$ with $\tilde{P}(-1)=0$ has a polynomial solution $R$.

Proof. First, let be

$$
P(w)=\frac{R\left(w^{2}\right)}{R(w)} \tilde{P}(w)
$$

and

$$
R(w)=\sum_{\ell=0}^{L} r_{\ell} w^{\ell} .
$$


We can choose $R$ as canonical solution of (7.2) so that $R(1)=1$, and Corollary 5.4 with $m=n=-1$ yields $\varphi_{-1}(t)=\sum_{\ell=0}^{L} r_{\ell} \tilde{\varphi}_{-1}(t-\ell)$, and therefore

$$
\sum_{k=0}^{\infty} \varphi_{-1}(t-k)=\sum_{\ell=0}^{L} r_{\ell} \sum_{k=0}^{\infty} \tilde{\varphi}_{-1}(t-\ell-k) .
$$

In view of $\tilde{P}(-1)=0$ Proposition 7.1 implies

$$
\sum_{k=0}^{\infty} \tilde{\varphi}_{-1}(t-\ell-k)=1 \quad(t>\tilde{N}+\ell-1) .
$$

Since $\ell \leq K$ and $\tilde{N}+K=N$, relation (7.1) follows from (7.4) and $R(1)=1$ together with (7.3).

Conversely, let (7.1) be satisfied, i.e.

$$
\sum_{k=0}^{N} \varphi_{-1}(t-k)=1 \quad(N-1<t<N+1)
$$

in view of (i). Substituting $t$ by $t+N$ and putting $N-k=\nu$, this relation is equal to (1.9), and in view of $\varphi_{-1}(t)=0$ for $t>N$ also to

$$
\sum_{\nu=0}^{\infty} \varphi_{-1}(t+\nu)=1 \quad(t<1)
$$

From this relation we can conclude as in the proof of [5: Theorem 3.3] that $P$ has form (7.2)

\section{Remark 7.3.}

1. It suffices in Theorem 7.2 that $\frac{R\left(w^{2}\right)}{R(w)}$ is a $w^{2}$-transformation of $\frac{1+w}{2}$, because all other factors of this quotient can be gathered up with $P$. Hence, the condition in Theorem 7.2 coincides with the necessary condition of [5: Theorem 3.3] for the existence of a Lebesgue-integrable solution of (1.1) with $\lambda=\frac{1}{2}$.

2. Let us mention that Assumptions 1.1 and 1.2 of Theorem 3.3 in [5] (and also of Theorem 2.1 there) are superfluous. Moreover, in [5: p. 63] there was used twice a wrong notation for the shift of the vector $\left(\zeta^{j}\right)_{j \geq 0}^{T} \mathbf{A}$.

3. Since $\varphi_{-1}(t+\nu)=0$ for $t+\nu>N$, we see first for $-1<t$ and $N+1 \leq \nu$ that (1.9) and (7.5) are equivalent, and second for $0<t$ and $\nu=N$ that relation (1.9) implies

$$
\sum_{\nu=0}^{N-1} \varphi_{-1}(t+\nu)=1 \quad(0<t<1) .
$$

Conversely, the last relation is equivalent to (1.9) for Lebesgue-integrable $\varphi_{-1}$ (and in particular for continuous ones). However, in the distributional case we cannot conclude from (7.6) to (1.9) without additional assumptions.

7.2 Linear independence. For stability questions of wavelet decompositions and subdivision schemes it is important that the shifts $\varphi_{-1}(t-\nu)(\nu \in \mathbb{Z})$ of eigenfunctions $\varphi_{-1}$ are linearly independent, i.e. that $\sum_{\nu} \sigma_{\nu} \varphi_{-1}(t-\nu)=0$ implies $\sigma_{\nu}=0$ for all $\nu$ (cf. $[15,16]$ ). By means of cyclic numbers under the mapping $f_{1}$ (cf. Definition 6.7), Theorem 2 in [16] can be simplified as follows: 
Proposition 7.4. The shifts of $\varphi_{-1}$ are linearly independent if and only if the characteristic polynomial $P$ satisfies the two conditions:

(a) $P$ has no symmetric zeros in $\mathbb{C}$.

(b) $P$ has no cyclic zeros under $f_{1}$ except possibly -1 .

In [16: Theorem 2] one finds instead of (b) the condition

$\left(\mathbf{b}^{\prime}\right)$ For any odd integer $k>1$ and a primitive $k^{\text {th }}$ root $\omega$ of unity, there exists an integer $d \geq 0$ such that $P\left(-\omega^{2^{d}}\right) \neq 0$.

However, it can be checked that conditions (b) and $\left(\mathrm{b}^{\prime}\right)$ are equivalent in view of (a). According to Proposition 6.2, Lemma 6.8 and Proposition 6.9 we obtain from Proposition 7.4 the

Corollary 7.5. The shifts of $\varphi_{-1}$ are linearly independent if and only if the characteristic polynomial $P$ has the form

$$
P(w)=\left(\frac{1+w}{2}\right)^{\alpha} \tilde{P}(w)
$$

where $\tilde{P}$ is a minimal characteristic polynomial, and $\alpha \in \mathbb{N}_{0}$.

A further immediate consequence of these assertions is [14: Theorem 5.3].

7.3 Infinite products. For a characteristic polynomial $P$ let us consider the infinite product

$$
S(w)=\prod_{j=0}^{\infty} \frac{1}{c_{0}} P\left(w^{2^{j}}\right)
$$

which converges for $|w|<1$ and satisfies $S(0)=1$, and which is closely connected with (4.6).

Proposition 7.6. The function (7.8) is rational if and only if $P$ is equivalent to the constant polynomial $\tilde{P}(w)=1$, i.e. if $P$ is of the form

$$
P(w)=\left(\frac{1+w}{2}\right)^{\alpha} \frac{p\left(w^{2}\right)}{p(w)}
$$

where $p$ is a polynomial with $p(0) p(1) \neq 0$, and $\alpha \in \mathbb{N}_{0}$. If $P$ has form (7.9) and if $p(0)=1$, then

$$
S(w)=\frac{1}{(1-w)^{\alpha} p(w)} .
$$

Proof. With the notations $R=\frac{1}{S}$ and $2^{\alpha}=\frac{1}{c_{0}}$ we obtain from (7.8) the equation

$$
2^{\alpha} P(w) R(w)=R\left(w^{2}\right),
$$

which can be interpreted as the basic functional equation (4.1) with $\tilde{P}=1$. Vice versa, $R$ is uniquely determined by (7.11) and $R(0)=1$ according to Proposition 4.2/1. 
Now, if the solution $R$ of (4.1) is rational, then Remark 4.5/2 yields $\alpha \in \mathbb{Z}$, Theorem 5.1 yields $P \sim 1$, and Proposition 6.2 representation (7.9) with $\alpha \in \mathbb{N}_{0}$, since $\tilde{P}=1$ is minimal. Finally, Corollary $6.3 / 2$ yields representation $(7.10)$ with $p(0)=1$ in view of $S(0)=1$.

Conversely, let $P \sim 1$. Then the polynomial $P$ has form (7.9) with $\alpha \in \mathbb{N}_{0}$ according to Proposition 6.2. Hence, with $R(w)=(1-w)^{\alpha} p(w)$ and $p(0)=1$ it follows $(7.11)$ and Proposition 4.2/1 together with $R(0)=1, \tilde{P}=1$ and $R=\frac{1}{S}$ yield (7.8)

In the case $P(w)=\frac{1+q w}{1+q}$ with $q \in \mathbb{C} \backslash\{-1\}$ product (7.8) reduces to

$$
S(w)=\prod_{j=0}^{\infty}\left(1+q w^{2^{j}}\right)=\sum_{k=0}^{\infty} q^{\nu(k)} w^{k} \quad(|w|<1)
$$

where $\nu(k)$ denotes the binary sum-of-digits function (cf. [4]). According to Proposition 7.6 function (7.12) is rational if and only if $P(w)=\frac{1+q w}{1+q} \sim 1$, and in view of Remark 6.6 we obtain for $q \neq-1$

Corollary 7.7. Only in the both cases $q=0$ and $q=1$ function (7.12) is rational.

However, Corollary 7.7 remains valid also in the excluded case $q=-1$, where $(7.12)$ is the generating function of the sign sequence $\varepsilon_{k}=(-1)^{\nu(k)}$.

\section{Reversed eigenfunctions}

For convenience we introduce

Definition 8.1. Two sequences $f_{n}$ and $p_{n}(n \in \mathbb{Z})$ and a function $F$ are termed an Appell triple, if they are connected with each other as in Proposition 2.1.

Note that an arbitrary generalized function $f_{m} \in \mathcal{F}_{m}$ with a fixed $m \in \mathbb{Z}$ generates an Appell triple, since the corresponding comparison sequence $f_{n}$ can be constructed by means of (1.4), the generating function $F$ by (2.10) and the Appell polynomials $p_{n}$ by (2.2). Examples 2.2, 2.3 and 2.4 can already be considered as examples for Appell triples. For two Appell triples $\left\{f_{n}, p_{n}, F\right\}$ and $\left\{f_{n}^{*}, p_{n}^{*}, F^{*}\right\}$ we can generalize [4: Proposition $4.2]$ :

Proposition 8.2. The following assertions are equivalent:

(a) $F^{*}(z)=e^{-s z} F(-z)$.

(b) $f_{n}(t)+(-1)^{n} f_{n}^{*}(s-t)=p_{n}(t)$.

(c) $p_{n}^{*}(t)=(-1)^{n} p_{n}(s-t)$.

Here $z \in \mathbb{C}, n \in \mathbb{Z}, t \in \mathbb{R}$ are arbitrary, whereas $s$ is a fixed non-negative number such that $f_{n}(t)=p_{n}(t)$ and $f_{n}^{*}(t)=p_{n}^{*}(t)$ for $t>s$.

Proof. We show that (a) $\Rightarrow(\mathrm{b})$, but first only for $n=-1$ and for Lebesgueintegrable functions. Since the supports of both functions $f_{-1}$ and $f_{-1}^{*}$ are contained in $[0, s]$, we have

$$
\int_{0}^{s} e^{-t z} f_{-1}^{*}(t) d t=\int_{0}^{\infty} e^{-t z} f_{-1}^{*}(t) d t=F^{*}(z)
$$


and

$$
\int_{0}^{s} e^{-t z} f_{-1}(s-t) d t=\int_{0}^{s} e^{(\tau-s) z} f_{-1}(\tau) d \tau=e^{-s z} \int_{0}^{\infty} e^{\tau z} f_{-1}(\tau) d \tau=e^{-s z} F(-z),
$$

so that (a) implies $f_{-1}^{*}(t)=f_{-1}(s-t)$, i.e. (b) is true for $n=-1$. By differentiation of the last equation it follows immediately that (b) is true for all $n<0$.

Next, we show by induction that (b) is also valid for $n \in \mathbb{N}_{0}$. Assume that (b) is valid for $n-1$ instead of $n$. Then, in view of $f_{n}^{\prime}=f_{n-1}, f_{n}^{* \prime}=f_{n-1}^{*}$ and $p_{n}^{\prime}=p_{n-1}$, we get by integration that

$$
f_{n}(t)+(-1)^{n} f_{n}^{*}(s-t)=p_{n}(t)+C_{n}
$$

where $C_{n}$ is a certain constant. For $t>s$ we obtain $C_{n}=0$ in view of $f_{n}(s)=p_{n}(s)$ and $f_{n}^{*}(s-t)=0$, so that (b) is also proved for $n \in \mathbb{N}_{0}$ and Lebesgue-integrable $f_{-1}$, $f_{-1}^{*}$.

If only $f_{m}$ is locally Lebesgue-integrable for a certain $m \in \mathbb{N}_{0}$, then by means of the representation

$$
F(z)=z^{m+1} \mathcal{L}\left\{f_{m}\right\}=z^{m+1} \int_{0}^{s} e^{-z t} f_{m}(t) d t+e^{-s z} \sum_{\nu=0}^{m} b_{\nu} z^{\nu}
$$

with $b_{\nu}$ from (2.7) and (2.8) it is possible to prove (a) $\Rightarrow(\mathrm{b})$ first in the case $n=m$. Afterwards the remaining steps can be repeated analogously.

Now we show $(\mathrm{b}) \Rightarrow(\mathrm{c})$. Substituting $t$ in (b) by $s-t$, and multiplying (b) with $(-1)^{n}$ we get

$$
(-1)^{n} f_{n}(s-t)+f_{n}^{*}(t)=(-1)^{n} p_{n}(s-t),
$$

so that comparison with (b) implies the polynomial relation (c).

Finally, it follows easily from

$$
e^{t z} F^{*}(z)=\sum_{n=0}^{\infty} p_{n}^{*}(t) z^{n}=\sum_{n=0}^{\infty}(-1)^{n} p_{n}(s-t) z^{n}=e^{(t-s) z} F(-z)
$$

that $(\mathrm{c}) \Rightarrow(\mathrm{a})$. Hence, the proposition is proved completely

Remark 8.3. Obviously, the $*$-operation is an involution, i.e. $F^{* *}=\left(F^{*}\right)^{*}=F$, $f^{* *}=f$ and $p_{n}^{* *}=p_{n}$ with the same $s$ as in Proposition 8.2.

Definition 8.4. Two Appell triples $\left\{f_{n}, p_{n}, F\right\}$ and $\left\{f_{n}^{*}, p_{n}^{*}, F^{*}\right\}$, and also their elements are called reversed, if they are connected with each other as in Proposition 8.2.

In particular, an Appell triple can be reversed to itself. A special example of a self-reversed Appell triple can be found in [3], where $f_{n}$ are the eigenfunctions $\varphi_{n}$ of a certain integral-functional equation. The former Example 2.4 with $K=0$ yields such a triple with $s=0$. In the case that the elements $f_{n}=\varphi_{n}$ of an Appell triple are eigenfunctions of (1.1), and that the elements $f_{n}^{*}=\varphi_{n}^{*}$ of a corresponding reversed Appell triple are eigenfunctions of the two-scale difference equation

$$
\lambda \varphi^{*}\left(\frac{t}{2}\right)=\sum_{\nu=0}^{N^{*}} c_{\nu}^{*} \varphi^{*}(t-\nu)
$$

with $c_{0}^{*} c_{N^{*}} \neq 0$, we also speak about reversed eigenfunctions $\varphi_{n}, \varphi_{n}^{*}$, and about reversed two-scale difference equations (1.1), (8.1). 
Proposition 8.5. The two-scale difference equations (1.1) and (8.1) are reversed if and only if the equation

$$
P^{*}(w)=w^{N} P\left(\frac{1}{w}\right)
$$

is satisfied, i.e. $N^{*}=N$ and $c_{\nu}^{*}=c_{N-\nu}$ for $\nu=0,1, \ldots, N$.

Proof. If both equations are reversed, then according to Proposition 8.2 the equation

$$
\phi^{*}(z)=e^{-s z} \phi(-z)
$$

must be satisfied with a certain constant $s \geq 0$. Replacing $z$ by $-2 z$ in (8.3), we have $\phi^{*}(-2 z)=e^{2 s z} \phi(2 z)$. Hence, by means of (2.19) and the analogous equation for $\phi^{*}$, we obtain

$$
e^{2 s z} \phi(2 z)=\phi^{*}(-2 z)=P^{*}\left(e^{z}\right) \phi^{*}(-z)=P^{*}\left(e^{z}\right) e^{s z} \phi(z)=P^{*}\left(e^{z}\right) e^{s z} \frac{1}{P\left(e^{-z}\right)} \phi(2 z),
$$

i.e.

$$
P^{*}\left(e^{z}\right)=e^{s z} P\left(e^{-z}\right) .
$$

Finally, with $w=e^{z}$ we get the equation

$$
P^{*}(w)=w^{s} P\left(\frac{1}{w}\right)
$$

which implies $s=N$ according to $c_{0}^{*} \neq 0$ and $c_{N} \neq 0$.

Conversely, if $P^{*}(w)=w^{N} P\left(\frac{1}{w}\right)$, then according to $(2.20)$, used both for $\phi$ and $\phi^{*}$, we have

$$
\phi^{*}(z)=\prod_{j=1}^{\infty} P^{*}\left(e^{-z / 2^{j}}\right)=\prod_{j=1}^{\infty} e^{-N z / 2^{j}} P\left(e^{z / 2^{j}}\right)=e^{-N z} \phi(-z),
$$

so that indeed equations (1.1) and (8.1) are reversed

The polynomial $P^{*}$, defined by (8.2), is called the reversed polynomial of $P$ (cf. [8]). As an application of Proposition 8.5 we see from (2.1) and (2.7) that (2.21) and (2.23) are reversed counterparts from each other. Further, for the eigenfunctions $\varphi_{n}^{*}$ of the two-scale difference equation

$$
\lambda \varphi\left(\frac{t}{2}\right)=\sum_{\nu=0}^{N} c_{N-\nu} \varphi(t-\nu)
$$

which is reversed to (1.1), and the corresponding Appell polynomials $p_{n}^{*}$ Proposition 8.2 yields:

Corollary 8.6. The eigenfunctions $\varphi_{n}$ and $\varphi_{n}^{*}(n \in \mathbb{Z})$ of the reversed equations (1.1) and (8.4) satisfy the relations

$$
\varphi_{n}(t)+(-1)^{n} \varphi_{n}^{*}(N-t)=p_{n}(t)
$$

and the corresponding Appell polynomials are connected by

$$
p_{n}^{*}(t)=(-1)^{n} p_{n}(N-t)
$$

for $t \in \mathbb{R}$.

Finally, we establish a connection between equivalent and reversed characteristic polynomials. For $(\tilde{P})^{*}$ we write for short $\tilde{P}^{*}$. 
Proposition 8.7. We have $P \sim \tilde{P}$ if and only if $P^{*} \sim \tilde{P}^{*}$. Moreover, if $R$ is the canonical solution of (4.1), then

$$
R^{*}(w)=(-1)^{\alpha} w^{N-\tilde{N}} R\left(\frac{1}{w}\right)
$$

is the canonical solution of

$$
2^{\alpha} P^{*}(w) R^{*}(w)=\tilde{P}^{*}(w) R^{*}\left(w^{2}\right)
$$

and vice versa.

Proof. From (4.1) with $\frac{1}{w}$ instead of $w$ we obtain after multiplication by $w^{2 N-\tilde{N}}$ that

$$
2^{\alpha} w^{N} P\left(\frac{1}{w}\right) w^{N-\tilde{N}} R\left(\frac{1}{w}\right)=w^{\tilde{N}} \tilde{P}\left(\frac{1}{w}\right) w^{2(N-\tilde{N})} R\left(\frac{1}{w^{2}}\right) .
$$

Using (8.2) and the notation

$$
R^{*}(w)=C w^{N-\tilde{N}} R\left(\frac{1}{w}\right)
$$

with a certain constant $C \neq 0$, the foregoing equation can be written as (8.8) and we see that $R^{*}$ is a rational solution of (8.8). In order to determine $C$ by means of (3.8), we consider

$$
\frac{R^{*}(w)}{(1-w)^{\alpha}}=(-1)^{\alpha} C w^{N-\tilde{N}-\alpha} \frac{R\left(\frac{1}{w}\right)}{\left(1-\frac{1}{w}\right)^{\alpha}}
$$

and for $w \rightarrow 1$ we find that $C=(-1)^{\alpha}$. The inversion follows in view of $R^{* *}=R$

Example 8.8 (Self-reversed canonical solutions). Let

$$
P(w)=\frac{1+w^{k}}{2} \tilde{P}(w)
$$

with $k \in \mathbb{N}$. After multiplication by $R(w)=\frac{1}{k}\left(1-w^{k}\right)$ this relation can be interpreted as basic functional equation (4.1) with $\alpha=1$ and $R$ as canonical solution. In view of $k=N-\tilde{N}$ we find from (8.7) the relation

$$
R^{*}(w)=-w^{k} \frac{1}{k}\left(1-\frac{1}{w^{k}}\right)=R(w)
$$

which matches with $P^{*}=\frac{1+w^{k}}{2} \tilde{P}^{*}$. 


\section{References}

[1] Antosik, P., Mikusiński, J. and R. Sikorski: Theory of Distributions. The Sequential Approach. Amsterdam: Elsevier Sci. Pub. Comp. 1973.

[2] Berg, L.: Operatorenrechnung II. Funktionentheoretische Methoden. Berlin: Dt. Verlag Wiss. 1974.

[3] Berg, L. and M. Krüppel: On the solution of an integral-functional equation with a parameter. Z. Anal. Anw. 17 (1998), 159 - 181.

[4] Berg, L. and M. Krüppel: De Rham's singular function, two-scale difference equations and Appell Polynomials. Result. Math. 38 (2000), 18 - 47.

[5] Berg, L. and G. Plonka: Compactly supported solutions of two-scale difference equations. Lin. Alg. Appl. 275-276 (1998), 49 - 75.

[6] Berg, L. and G. Plonka: Spectral properties of two-slanted matrices. Result. Math. 35 (1999), $201-215$.

[7] Berg, L. and G. Plonka: Some notes on two-scale difference equations. In: Functional Equations and Inequalities (Mathematics and Its Applications: Vol. 518; ed.: Th. M. Rassias). Dodrecht-Boston-London: Kluwer Acad. Publ. 2000, pp. $7-29$.

[8] Boas, R. P. and R. C. Buck: Polynomial expansions of Analytic Functions. Berlin Göttingen - Heidelberg: Springer-Verlag 1958.

[9] Cavaretta, A. S., Dahmen, W. and C. A. Micchelli: Stationary Subdivision (Memoirs of the Amer. Math. Soc.: Vol. 453). Providence: Amer. Math. Soc. 1991.

[10] Chui, C. K.: An Introduction to Wavelets. Boston et al.: Acad. Press 1992.

[11] Daubechies, I.: Ten Lectures on Wavelets. Philadelphia: SIAM 1992.

[12] Daubechies, I. and J. Lagarias: Two-scale difference equations I. Existence and global regularity of solutions. SIAM J. Math. Anal. 22 (1991), 1388 - 1410.

[13] Daubechies, I. and J. Lagarias: Two-scale difference equations II. Local regularity, infinite products of matrices and fractals. SIAM J. Math. Anal. 23 (1992), 1031 - 1079.

[14] Jia, R. Q.: Subdivision schemes in $L_{p}$ spaces. Adv. Comp. Math. 3 (1995), 309 - 341.

[15] Jia, R. Q. and C. A. Micchelli: Using the refinement equations for the construction of prewavelets II: Powers of two. In: Curves and Surfaces (eds.: P.-J. Laurent, A. Le Méhanté and L. L. Schumaker). Boston et al.: Acad. Press 1991, pp. $209-246$.

[16] Jia, R. Q. and J. Wang: Stability and linear indepedence associated with wavelet decompositions. Proc. Amer. Math. Soc. 117 (1993), 1115 - 1124.

[17] Lawton, W., Lee, S. L. and Z. Chen: Characterization of compactly supported refinable splines. Adv. Comput. Math. 3 (1995), 137 - 145.

[18] Maeß, G.: Vorlesungen über numerische Mathematik II. Berlin: Akademie-Verlag 1988.

[19] Micchelli, C. A.: Using the refinement equation for the construction of pre-wavelets. Numer. Algorithms 1 (1991), 75 - 116.

[20] Schoenberg, I. J.: Cardinal Spline Interpolation. Philadelphia: SIAM 1973.

[21] Targonski, G.: Topics in Iteration Theory. Göttingen - Zürich: Vanderhoeck \& Ruprecht 1981.

Received 03.07.2000; in revised form 27.02.2001 